\title{
Transfer of near infrared spectrometric models for silage crude protein detection between different instruments
}

\author{
X. Liu, L. J. Han, ${ }^{1}$ and Z. L. Yang \\ College of Engineering, China Agricultural University, Beijing 100083, China
}

\begin{abstract}
This study was undertaken to distinguish different collected spectra from near infrared reflectance spectroscopy (NIRS) for silages using different configurations and types of NIRS, and how well the different techniques for transferring NIRS calibrations perform for silage crude protein detection. In the study, 2 Fourier transform instruments and 1 scanning grating instrument were involved. Five correction and transfer methods were tested and evaluated: slope/bias, local centering, orthogonal signal correction, direct standardization, and piecewise direct standardization. We concluded that the spectra obtained with 3 instruments were different and not solely due to the differences in offset. All of the methods for calibration transferring between 2 Fourier transform instruments and 1 Fourier transform instrument versus 1 scanning grating instrument could improve the predictions, but not all of the results could be accepted. The slope/bias, orthogonal signal correction, and local centering techniques were successful for calibration transferring of 2 Fourier transform instruments, considering their good performance. The best result was given for orthogonal signal correction ahead of the other 4 techniques for transferring calibrations between instruments of Fourier transform and scanning grating, and it was evaluated as moderately useful.
\end{abstract}

Key words: calibration transfer, near infrared reflectance spectroscopy, silage, crude protein

\section{INTRODUCTION}

Near infrared reflectance spectroscopy (NIRS) has become a commonly used and increasingly analytical method in many fields during recent decades due to its characteristics as being fast, cost efficient, nondestructive to samples, and not involving use of hazardous chemicals. It is also known that NIRS calibrations generally involve hundreds of samples and considerable

Received March 21, 2011.

Accepted August 1, 2011.

${ }^{1}$ Corresponding author: hanlj@cau.edu.cn time and costs for sample collection and wet chemical analysis. However, sometimes the developed calibrations are not very useful. They would be inapplicable when the spectra of samples for predicting are collected on a second instrument, or if, for example, the instrument is old or in need of repair or upgrading for critical parts, if physical modifications of the calibration or prediction samples are made, or if a change of work circumstance occurs. To solve these problems, transferring or correcting the existing near-infrared spectrometric models is necessary to avoid those time-consuming recalibration procedures and to apply NIRS to industrial process control economically. Several different methods have been proposed for calibration transfer (Wang et al., 1991; Blank et al., 1996; Bouveresse et al., 1996; Lorber et al., 1996; Wold et al., 1998; Koehler et al., 2000) based on many steps. Calibrations can be improved by simple correction of the $Y$ value, multivariate modification of sample spectra, or complicated operation both on spectra and chemical values of samples.

Over the last few years, NIRS technology has been introduced in corn and corn silage evaluation with good results (Moe and Carr, 1985; Melchinger et al., 1986; De Boever et al., 1997; Cozzolino et al., 2001; Sørensen, 2004). Previously, we have studied the feasibility of NIRS for predicting chemical parameters and digestibility of silage samples, and many robust calibrations have been established (Liu and Han, 2006; Liu et al., 2008). This study was designed to discover the differences of the collected spectra from different NIRS instruments for silages and to evaluate calibration transfer of different techniques: slope/bias correction (SB), local centering (LC), orthogonal signal correction (OSC), direct standardization (DS), and piecewise direct standardization (PDS) for existing near-infrared spectrometric models for CP detection of silage. All of these methods were applied separately to 2 Fourier transform instruments and 1 scanning grating instrument.

\section{MATERIALS AND METHODS}

\section{Silage Samples and CP Analysis}

This study involved 141 silage samples obtained from cattle farms and laboratories of 6 provinces (Hebei, 
Table 1. Instruments used in this study

\begin{tabular}{|c|c|c|c|c|}
\hline Manufacturer & Configuration & Type & Detector $^{1}$ & Label \\
\hline Thermo Nicolet Corp. (Madison, WI) & Antaris; integrating sphere accessory & Fourier transform & InAs & Slave 1 \\
\hline Foss A/S (Hillerød, Denmark) & NIRSystems 6500 ; rapid content analyzer & Scanning grating & $\mathrm{PbS}$ & Slave 2 \\
\hline
\end{tabular}

${ }^{1} \mathrm{InAs}=$ indium arsenic; $\mathrm{PbS}=$ lead sulfide.

Zhejiang, Jiangsu, Jilin, Heilongjiang, and Shanxi) in China. Among these samples, 67 were whole plant corn silages, 58 were corn stalk silages, and the total number of samples of rice, wheat, and sorghum silage was 16 . The samples were ensiled with or without silage additives (Lactobacillus, enzyme, molasses and formic acid) by different techniques (ensiling with silos, polyethylene bags, and barrels). All of the collected silage samples had a particle size between 2 and $10 \mathrm{~cm}$. They were dried in a forced-air drier at $65^{\circ} \mathrm{C}$ for $48 \mathrm{~h}$ on the day of collection, and then milled to pass a 1-mm screen for NIRS scanning and wet chemical analysis.

Crude protein content analysis was carried out concurrently with the scanning according to official methods (AOAC, 2001). The chemical analyses were done in duplicate, and expressed on a dry weight basis.

All of the samples were sorted by the CP composition, and 1 of 4 removed as a validation sample. Therefore, the data set was divided into a calibration set of 106 samples and a validation set of 35 samples according to their composition. Then, the silages in the calibration set were sorted by the composition and 1 of 3 removed as a standardization sample. Thus, a standardization set of 30 samples was selected according to the suggestions of Shenk and Westerhaus (1991, 1993).

\section{NIRS Instruments and Calibration}

Three NIRS systems were involved in this study (Table 1), 2 of which were Fourier transform instruments from PerkinElmer Inc. (Seattle, WA; Spectrum One NTS) and Thermo Nicolet Corp. (Madison, WI; Antaris), and the third was a scanning grating instrument from Foss A/S (Hillerød, Denmark; NIRSystems 6500). The Fourier transform instrument from PerkinElmer Inc. was used as the master instrument, and the instrument from Thermo Nicolet Corp. was studied as slave 1. The Fourier transform and scanning grating instruments were equipped with indium arsenide (InAs) and lead sulfide $(\mathrm{PbS})$ detectors, respectively. All of the spectra were measured in cups with a quartz window. On the Fourier transform instruments, the spectra of calibration and additional validation samples were acquired in the wave number range 10,000 to $4000 \mathrm{~cm}^{-1}$ and the resolution was $8 \mathrm{~cm}^{-1}$. Spectra of additional validation samples were also collected over the wavelength range
1,100 to $2,500 \mathrm{~nm}$ with 2 -nm digital resolution on the scanning grating instrument from Foss $\mathrm{A} / \mathrm{S}$, and it was studied as the slave 2 instrument. The standardization samples were scanned on each instrument, respectively. The number of scans per spectra was set to 32 and scanning was performed 3 times per sample; the average of the 3 scans was recorded as $\log 1 / R$, where $R$ is the reflectance ratio of light.

The main reason for the selection of the master instrument was that robust partial least-square (PLS) calibration models for many parameters based on the Fourier transform instrument from PerkinElmer Inc. have been developed in the past (Liu and Han, 2006; Liu et al., 2008). The NIRS calibrations were developed by means of PLS regression on full-scan spectra on the master instrument. In the current study, CP was chosen to carry out calibration transfer and the goal was to apply it to other parameters later. The following mathematical treatments were applied separately and simultaneously: smoothing, derivative, standard normal variate, and multiplicative scatter correction, and the best pretreatment, resulting in the lowest root mean square error of prediction (RMSEP), was chosen. The best number of PLS terms was determined by considering the lowest RMSEP with the fewest terms.

\section{Calibration Transfer and Correction Techniques}

Calibration transfers were performed, in turn, between 2 Fourier transform instruments (master and slave 1), 1 Fourier transform instrument (master), and 1 scanning grating instrument (slave 2). The following 5 correction and transfer methods were used in this study: SB, LC, OSC, DS, and PDS.

The SB technique is a univariate approach that is widely used for correcting $Y$ values by the standardization samples measured on both instruments (Bouveresse et al., 1996; Fearn, 2001). The spectra of standardization samples measured on both instruments were first predicted by the calibration model developed on the master system. A linear regression was then fitted between the 2 property predictions with orthogonal least squares, and the slope and bias correction factors were calculated. The predicted values of the validation samples measured on the slave instrument were corrected by the equation 


$$
y_{\text {corr }}=\text { bias }+ \text { slope } \times y,
$$

where $y$ is the predicted value of validation samples measured on the slave instrument and $y_{\text {corr }}$ is the corrected value of validation samples measured on the slave instrument.

In the LC method, the spectral matrix of calibration samples measured on master instruments and validation samples measured on slave instruments were local centered first. In this case, all data sets (calibration, as well as validation sets) were centered with respect to the mean vector of the transfer samples for the respective instruments. The $\mathbf{y}$ vector was also centered. A new calibration was then developed with the centered $\mathbf{y}$ and spectral values of calibration samples measured on master instruments, and the local centered spectra of validation samples were predicted (Lorber et al., 1996; Leion et al., 2005).

Both DS and PDS techniques were mathematical models that aimed to find a matrix and a background vector that transformed a spectrum measured on a slave instrument into what it should have looked like being measured on the master instrument. A transformation matrix $\mathbf{F}$ was computed by multivariate regression based on the spectral response of the standardization samples measured on both instruments. Then, it could be used for the correction of the spectral matrix of the validation samples measured on the slave instrument. For the DS method, each wavelength of the master instrument was related to all wavelengths of the slave instrument simultaneously, whereas for the PDS method, spectral intensities at a certain wavelength on the master instrument were related to the wavelengths located in a spectral window around the same wavelength on the slave instrument (Wang et al., 1991; Feudale et al., 2002). In this study, the effect of different numbers of channels in the moving spectral window was also examined.

The OSC was designed to remove irrespective vectors of spectral matrix that were orthogonal to $y$ values (Sjöblom et al., 1998; Wold et al., 1998). First the spectra matrix $\mathbf{X}$ of the standardization set, which consisted of $X_{m}$ block for the master instrument and $X_{s}$ block for the slave instrument, was global centered:

$$
\mathbf{X}=\left[X_{m}, X_{s}\right]-\frac{\left(\bar{x}_{m}+\bar{x}_{s}\right)}{2},
$$

where $\bar{x}_{m}$ and $\bar{x}_{s}$ are the average spectrum of standardization sets measured on the master instrument and slave instrument, respectively. The $\mathbf{y}$ vector was always global centered. Then the matrix $\mathbf{X}$ was orthogonalized to $\mathbf{y}$ until a stable $\mathbf{T}$ vector was gained, which was a good descriptor of part of matrix $\mathbf{X}$ that was orthogonal to $\mathbf{y}$. Matrix $\mathbf{W}$ and loading $\mathrm{P}$ were computed:

$$
\begin{gathered}
\mathrm{W}=\mathbf{X}^{-1} \mathbf{T}, \\
\mathrm{P}=\mathbf{X} \mathbf{T}^{\mathbf{T}} /\left(\mathbf{T}^{\mathbf{T}} \mathbf{T}\right) .
\end{gathered}
$$

The aim spectral matrix $\mathbf{X}$ was then corrected by using the matrix $\mathbf{W}$ and loading $\mathbf{P}$ :

$$
\mathrm{X}_{\mathrm{osc}}=\mathbf{X}-\mathbf{X W P}^{\mathrm{T}} \text {. }
$$

\section{Statistical Analysis and Multivariate Evaluation}

All of the raw spectra data were imported into Unscrambler software (CAMO Inc., Oslo, Norway). Mathematical treatments and calibrations were performed in this software. Calibration transfer calculations were carried out in Matlab 7.0 (The MathWorks Inc., Natick, MA). The PLS Toolbox 2.0 (Eigenvector Research Inc., Manson, WA) was used for DS, PDS, and OSC corrections.

Preliminary validation data evaluation was performed using spectral leverage versus student residuals of actual value plot to detect the outlier samples. Samples with high leverage and high residue were excluded (Hein et al., 2009).

For the spectra measured with different instrument resolutions, the spectra measured on the master instrument were chosen to be the references and spectra measured on slave instruments were converted to match. The method of cubic spline interpolation and not-aknot end conditions were used in the conversions.

The results of transfer were evaluated by comparing the prediction errors obtained using the model developed on the master instrument. The values of RMSEP and mean bias were used for estimating the prediction error. Meanwhile, the average diversity of spectra (ADS) and $\mathrm{P}_{\mathrm{ADS}}$ were designed for the difference of spectrum data between 2 instruments before or after transferring for the efficiency evaluation of a certain method. The calibration transfer method resulting in the lowest values of RMSEP, bias, and ADS and the highest value of $\mathrm{P}_{\text {ADS }}$ was considered to be the most active one. The prediction errors for the slave instruments were sought to be as low as those for the master system. And, in general, RMSEP and bias values directly indicated the prediction error, so they were more important than ADS and $\mathrm{P}_{\mathrm{ADS}}$ values. The NIRS predictions were further evaluated by the ratio of the standard deviation of the values in the validation set to the RMSEP, termed the relative prediction deviation (RPD) statistic. A guideline was suggested for describing the performance 
LIU ET AL.

Table 2. Statistics for the calibration, validation, and standardization sets

\begin{tabular}{lccccc}
\hline $\begin{array}{l}\text { Sample } \\
\text { set }\end{array}$ & $\begin{array}{c}\text { Number of } \\
\text { samples }\end{array}$ & $\begin{array}{c}\text { Maximum } \\
(\mathrm{g} / \mathrm{kg} \text { of } \mathrm{DM})\end{array}$ & $\begin{array}{c}\text { Minimum } \\
(\mathrm{g} / \mathrm{kg} \text { of } \mathrm{DM})\end{array}$ & $\begin{array}{c}\text { Mean } \\
(\mathrm{g} / \mathrm{kg} \text { of DM })\end{array}$ & $\mathrm{SD}$ \\
\hline Calibration & 106 & 115.48 & 48.38 & 83.46 & 15.99 \\
Validation & 34 & 105.73 & 48.69 & 82.18 & 15.55 \\
Standard & 30 & 115.48 & 48.38 & 83.63 & 16.97 \\
\hline
\end{tabular}

of predictions for agricultural applications as follows: excellent predictions: RPD >4; successful predictions: RPD 2.25 to 4 ; and moderately useful RPD: 1.75 to 2.25 (Murray, 1993; Williams and Sobering, 1993; Malley et al., 2004). Also, it has been shown that an RPD of approximately 1.50 indicates that NIRS can be used as an initial screening tool (Schimleck et al., 2003).

The RMSEP and bias were defined as

$$
\begin{aligned}
\text { RMSEP } & =\sqrt{\frac{\sum_{i=1}^{n_{v}}\left(y_{i}^{r e f}-\hat{y}_{i v}^{N I R}\right)^{2}}{n_{v}},} \\
\text { bias } & =\frac{\sum_{i=1}^{n_{v}}\left(y_{i}^{r e f}-\hat{y}_{i v}^{N I R}\right)}{n_{v}},
\end{aligned}
$$

where $y_{i}^{\text {ref }}$ is the wet chemical value, $\hat{y}_{i v}^{N I R}$ is the NIRS predicted value, and $n_{v}$ is the sample number of validation set.

The ADS and $\mathrm{P}_{\mathrm{ADS}}$ were calculated as

$$
\begin{gathered}
\mathrm{ADS}=\frac{1}{n_{v}} \sum_{i=1}^{n_{v}} \sqrt{\frac{1}{N} \sum_{\lambda=1}^{N}\left|S_{2 \lambda}^{i}-S_{1 \lambda}^{i}\right|}, \\
\mathrm{P}_{\mathrm{ADS}}(\%)=\frac{A D S_{\text {untransferred }}^{2}-A D S_{\text {transferred }}^{2}}{A D S_{\text {untransferred }}^{2}} \times 100 \%,
\end{gathered}
$$

where $N$ is the wavelength number, $S_{2 \lambda}^{i}$ and $S_{1 \lambda}^{i}$ are the absorbency values of wavelength $\lambda$ of the standard sample for 2 instruments, $A D S_{\text {untransferred }}$ is the ADS before transferring, and $A D S_{\text {transferred }}$ is the ADS after transferring.

\section{RESULTS AND DISCUSSION}

\section{Chemical and Spectroscopic Characterization}

The CP content of silage samples in the calibration set varied widely and was good for calibration. For the validation set, an anomalous sample was detected and excluded, examining the student residuals and leverage value scatter plot. The maximum, minimum, mean value, and standard deviation of the chemical parameters for the calibration set, validation set, and standardization set are summarized in Table 2 .

The raw mean spectra of the standardization set samples measured with 3 NIRS instruments of different configurations and types are shown in Figure 1.

It has been suggested that no significant differences in wavelength scale could be detected (Leion et al., 2005). Therefore, the spectra of scanning grating instrument were recalculated from wavelength to wave number range for compatibility. It was clear that the spectra obtained with the 3 instruments were different, and the differences of spectra between the 2 Fourier transform instruments (master and slave 1) were smaller than the differences of spectra between the Fourier transform instrument and scanning grating instrument (master and slave 2, slave 1 and slave 2). For master and slave 1 instruments, the difference was mostly wavelength shift; large differences in spectral intensity in the range of 5,400 to $4,000 \mathrm{~cm}^{-1}$ were found and maximally 0.12 in absorbance, whereas wavelength shifts and variable intensity shifts of spectra were obviously detected for master and slave 2, and slave 1 and slave 2 instruments. Complicated reasons for the differences may exist (i.e., different prismatic mode, different signal-to-noise ratio, response differences with different detectors, or a multiplicative effect or combination of many factors; Fearn, 2001). Whatever the reason, all of the present differences would cause significant deviations in the predictions if they were not disposed of by transfer techniques. The result of average diversity of spectra for 30 standard samples proved that the ADS was 0.16 and 0.31 for master versus slave 1 instruments, and master versus slave 2 instruments, respectively.

A principal component analysis (PCA) calibration was developed for the validation set of 3 instruments and the wave number range 9,090 to $4,000 \mathrm{~cm}^{-1}$ was used. Figure 2 shows the PCA score scatter plot for the first and third principal components ( $\mathrm{PC} 1$ versus PC3). Note that spectrum was grouped into clusters corresponding to 3 different instruments.

\section{Calibration and Predictions Before Transfer}

The resulted best PLS terms for calibration of CP was 7 and the raw spectral data was pretreated with 


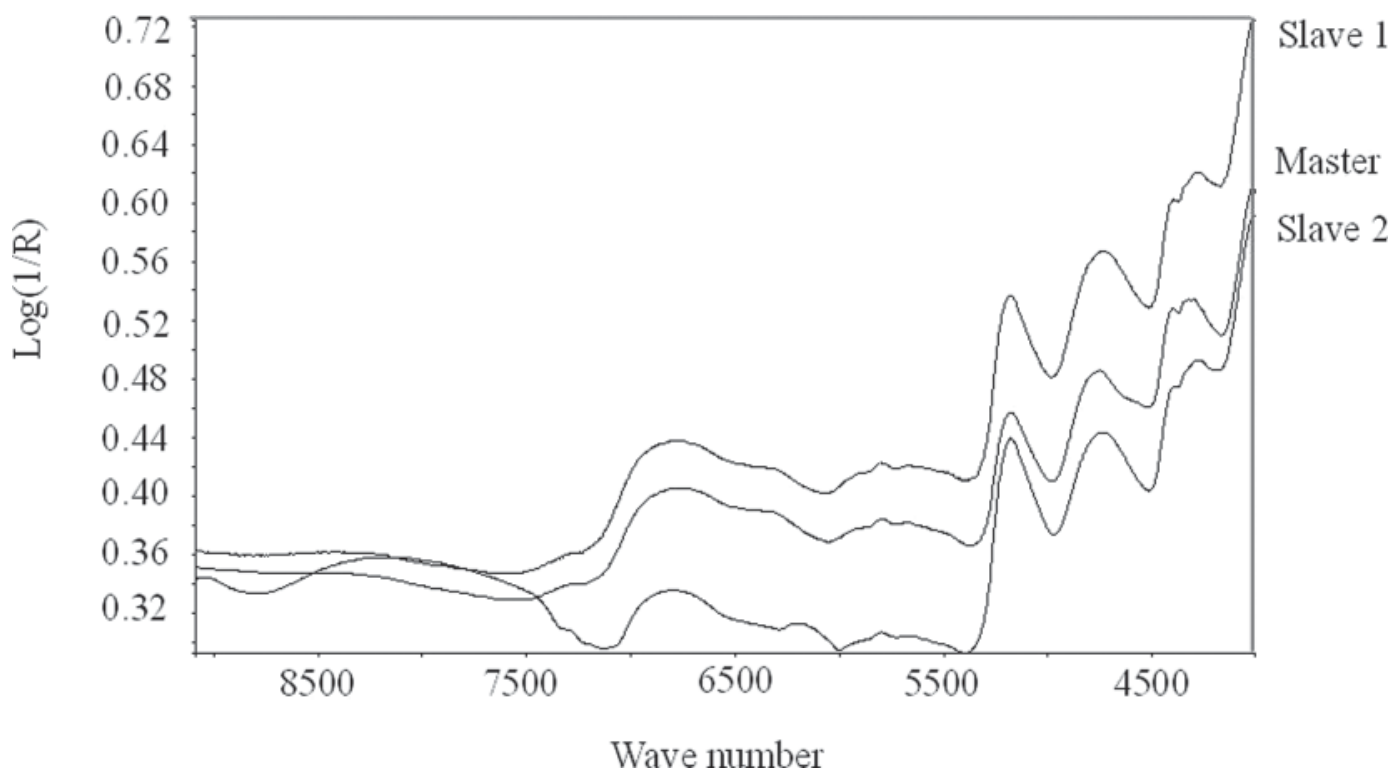

Figure 1. Mean spectra of standardization set samples measured with 3 near infrared (NIR) instruments. Slave $1=$ Antaris (Thermo Nicolet Corp., Madison, WI); master = Spectrum One NTS (PerkinElmer Inc., Seattle, WA); slave $2=$ NIRSystems 6500 (Foss A/S, Hillerød, Denmark); $\mathrm{R}=$ reflectance ratio of light.

smooth and first derivative using the Savitzky-Golay method (Savitzky and Golay, 1964). The correlation coefficient of calibration ( $\mathrm{r}$ ) and the root mean square error of calibration for the model established on the master instrument were 0.90 and $5.16 \mathrm{~g} / \mathrm{kg}$ of $\mathrm{DM}$, respectively (Figure 3).

For 3 NIRS instruments, the prediction error for validation set was studied using this calibration developed on the master instrument before calibration transfer. Prediction values of RMSEP, mean bias, and RPD are shown in Figure 4.

Good values of RMSEP and bias were achieved for the master instrument. The RMSEP and bias were $4.29 \mathrm{~g} / \mathrm{kg}$ of $\mathrm{DM}$ and 0.14 , respectively. The values of RMSEP and bias for the prediction obtained using the 2 slave instruments were not at this stage ideal - they were both much higher than the master instrument. The RMSEP and bias were $12.49 \mathrm{~g} / \mathrm{kg}$ of DM and 11.17 for the slave 1 instrument, and $22.28 \mathrm{~g} / \mathrm{kg}$ of $\mathrm{DM}$ and 6.75 for the slave 2 instrument. The performance of predictions for the 3 instruments were farther evaluated by the RPD value, and it was successful for the master instrument, with a high RPD value of 3.62 . The RPD values for both slave instruments were all less than 1.50, which demonstrated that the predictions could not be used as an initial screening tool yet. The prediction errors were in accordance with the result of the ADS given before. Therefore, calibration transfer was necessary for both of the 2 slave instruments.

\section{Calibration Transfer Between 2 Fourier Transform Instruments}

The standardization set of 30 samples was used for the SB technique to establish a linear equation based on the predictions obtained for both instruments. Then, the slope and bias (intercept) of the regression line were gained as 1.04 and 8.95 for later SB correction. For the LC method, to study the accuracy attenuation of the NIRS model developed on the centered spectrum and chemical values, prediction was studied on a validation set of 34 centered spectra obtained on the master instrument. The RMSEP value and bias were $4.49 \mathrm{~g} /$ $\mathrm{kg}$ of DM and 1.34, respectively, which was comparable with prediction errors of uncentered models and the validation set. The accuracy attenuation of the NIRS model developed on the corrected spectrum of the calibration set by the OSC technique was also considered. Results showed that the best number of the PLS term of corrected calibration was also 7 . The values of the correlation coefficient and RMSEC were 0.90 and 5.24 $\mathrm{g} / \mathrm{kg}$ of DM, respectively, which were similar to the calibration results before OSC correction. For the PDS method, the efficiency of different window sizes was studied and the optimum window size of 7 with lowest RMSEP and bias was ultimately chosen.

In Table 3, the values of RMSEP, bias for predictions, and ADS, and the $\mathrm{P}_{\mathrm{ADS}}$ value achieved for the slave 1 instrument after correction with different methods is 


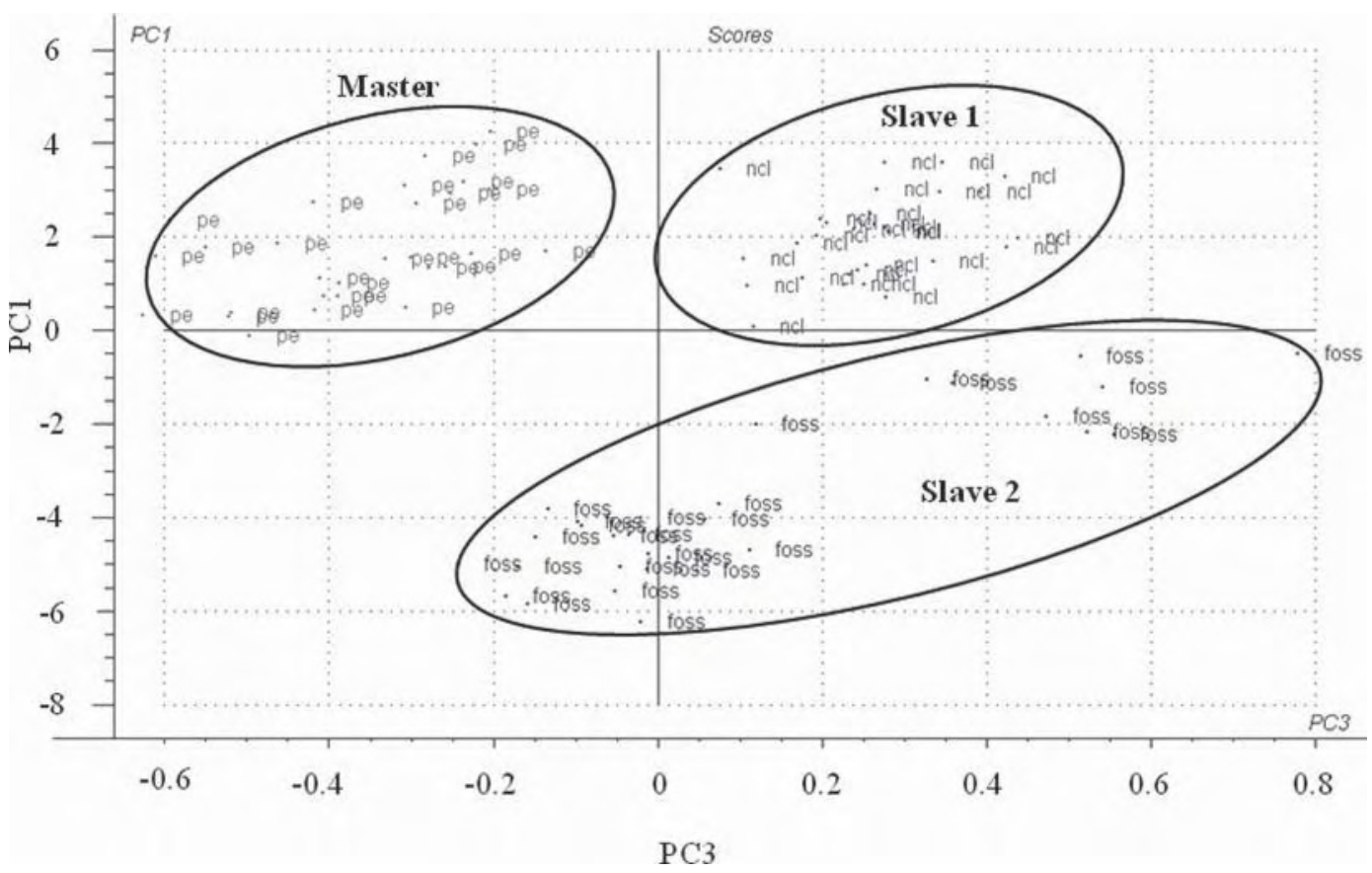

Figure 2. Principal component analysis score scatter plot for validation set with the wave number range of 9,090 to $4,000 \mathrm{~cm}^{-1}$. Master $=$ Spectrum One NTS [PerkinElmer Inc. (pe), Seattle, WA]; slave $1=$ Antaris [Thermo Nicolet Corp. (ncl), Madison, WI]; slave $2=$ NIRSystems 6500 [Foss A/S (foss), Hillerød, Denmark]; PC1 and PC3 are principal components 1 and 3, respectively.

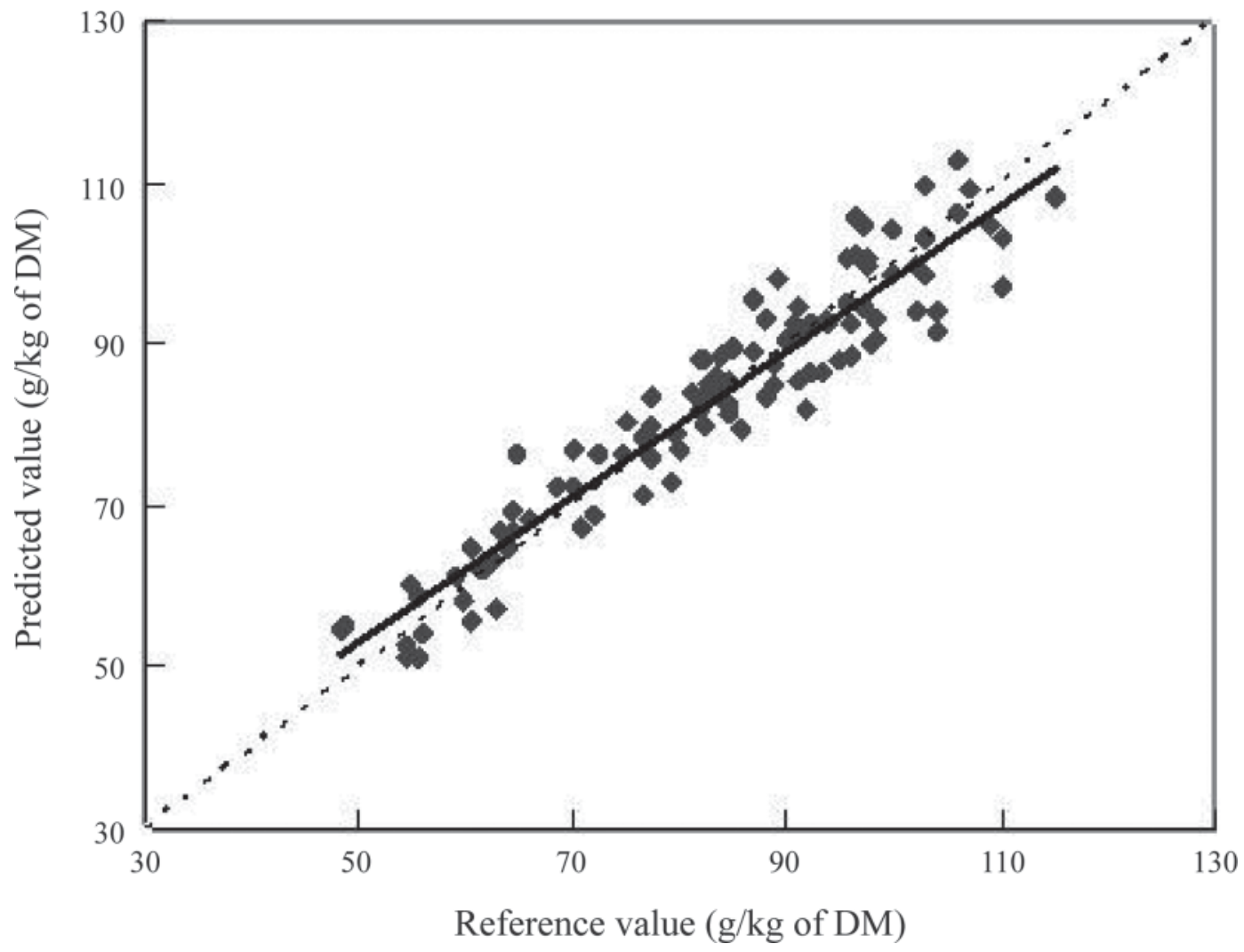

Figure 3. Calibration of CP established on the master instrument (Spectrum One NTS; PerkinElmer Inc., Seattle, WA) for 106 samples (reference values vs. predicted values). 


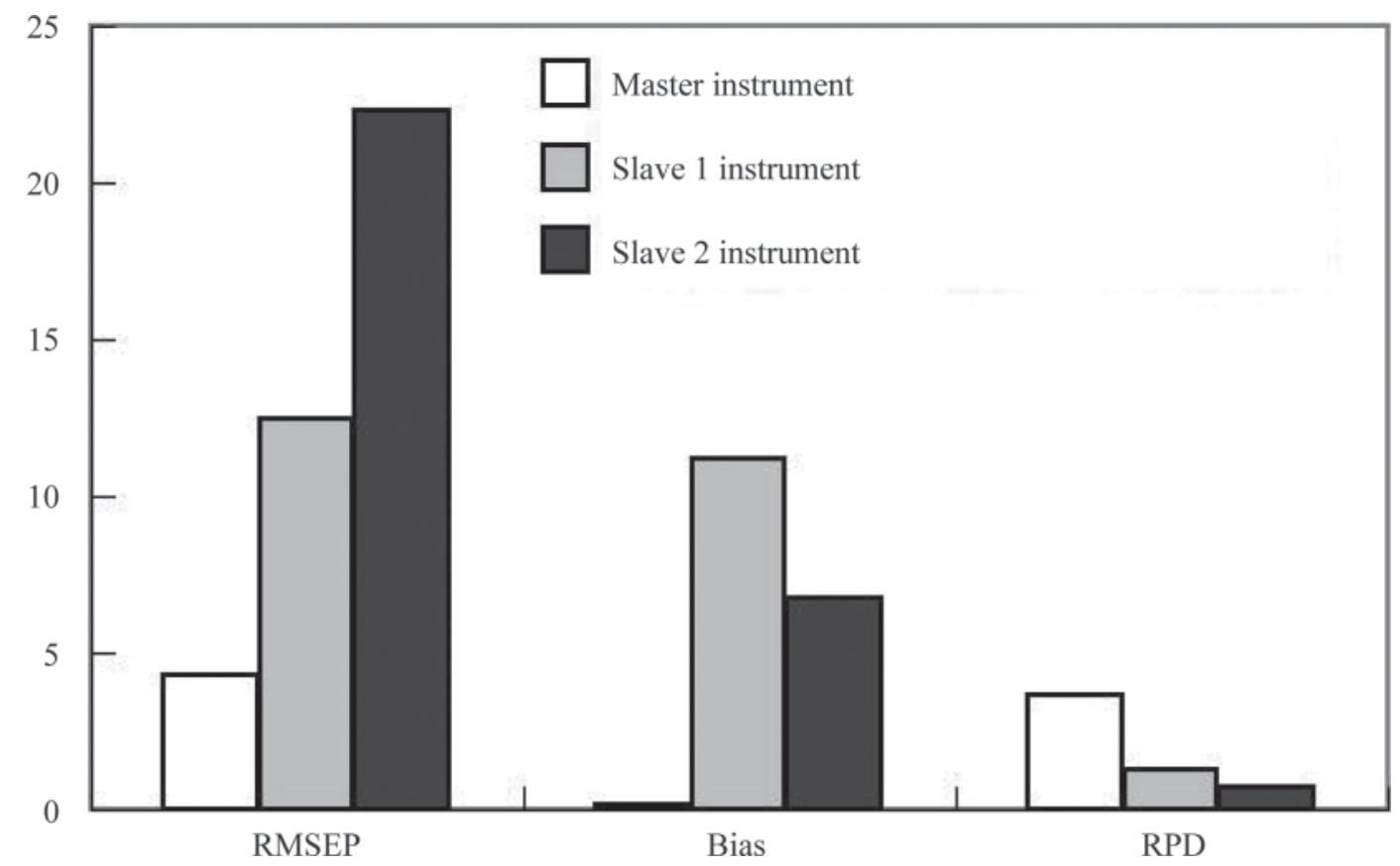

Figure 4. Prediction error for validation set obtained using the model developed on the master instrument (Spectrum One NTS; PerkinElmer Inc., Seattle, WA) before calibration transfer. Slave 1 = Antaris (Thermo Nicolet Corp., Madison, WI); slave $2=$ NIRSystems 6500 (Foss A/S, Hillerød, Denmark); RMSEP = root mean square error of prediction; RPD = relative prediction deviation.

shown. As the SB technique is only correct for $Y$ values with the slope and bias correction factors, the values of spectra diversity are absent.

Results showed that all the methods were active for NIRS calibration transferring between 2 Fourier transform instruments (master and slave 1). The RMSEP values after transfer were 5.65, 5.74, 5.80, 7.03, and $8.73 \mathrm{~g} / \mathrm{kg}$ of $\mathrm{DM}$, and bias values were $-0.43,0.18$, $1.50,-0.90$, and -0.97 for SB, OSC, LC, DS, and PDS methods, respectively. Results with a non-transfer technique were compared (RMSEP: $12.49 \mathrm{~g} / \mathrm{kg}$ of DM, bias: 11.17). The prediction errors derived by these 5 methods were all decreased for the slave 1 instrument. Accordingly, the spectral diversity was also decreased to different degrees after being transferred by OSC, LC, DS, and PDS methods. Good results were gained for the OSC method for which the ADS and $\mathrm{P}_{\mathrm{ADS}}$ values were 0.07 and $80.86 \%$, respectively. It was evident that SB and OSC corrections almost worked equally well for transferring of the present NIRS calibration for silage $\mathrm{CP}$ detection and no significant differences between their results were observed $(P>0.05)$. It was explained before that the SB technique simply corrected $Y$ values by the standardization samples measured on both instruments, and the OSC was designed to remove irrelevant vectors of the spectral matrix that are orthogonal to $Y$ values by more complicated arithmetic and programs. Therefore, the SB technique should be preferred as the most simple and robust approach, considering its good performance and maneuverability. Moderate function was obtained for the LC technique. Although comparable RMSEP values were obtained with the SB and OSC techniques, but their bias values were a little higher than that of the SB method, and the ADS value

Table 3. Results of the slave 1 (Antaris; Thermo Nicolet Corp., Madison, WI) instrument after being transferred by different methods

\begin{tabular}{lccrrrr}
\hline & \multicolumn{7}{c}{ Method $^{2}$} \\
\cline { 2 - 7 } Parameter $^{1}$ & None & SB & OSC & \multicolumn{1}{c}{ LC } & \multicolumn{1}{c}{ DS } & \multicolumn{1}{c}{ PDS } \\
\hline RMSEP & 12.49 & 5.65 & 5.74 & 5.80 & 7.03 & 8.73 \\
Bias & 11.17 & -0.43 & 0.18 & 1.50 & -0.90 & -0.97 \\
ADS & 0.16 & - & 0.07 & 0.12 & 0.14 & 0.13 \\
$\mathrm{P}_{\text {ADS }} / \%$ & - & - & 80.86 & 43.75 & 23.44 & 33.98 \\
\hline
\end{tabular}

${ }^{1} \mathrm{RMSEP}=$ root mean square error of prediction; ADS = average diversity of spectra; $\mathrm{P}=$ loading. ${ }^{2} \mathrm{SB}=$ slope/bias correction; OSC $=$ orthogonal signal correction; LC = local centering; DS = direct standardization; PDS = piecewise direct standardization 
was a little higher than that of the OSC method. For the techniques of DS and PDS, they gave a similar efficiency, which was less than the other 3 methods.

Statistical analysis also showed that no significant difference could be discovered for the predictions achieved from the master instrument and the ones achieved from the slave 1 instrument after being transferred by the SB and OSC methods $(P>0.05)$. It was suggested that prediction errors for the slave 1 instruments could be sought to be as low as those for the master system by transfer technique of SB and OSC.

Predictions after being transferred by different methods were further evaluated by the RPD value (Figure $5)$. It was obvious that the RPD values of SB, OSC, and LC methods were all higher than 2.25, which demonstrated that calibration transfer between 2 Fourier transform instruments functioning by these 3 techniques were all successful. Predictions transferred by DS and PDS were moderately useful, with both of the RPD values between 1.75 and 2.25.

\section{Calibration Transfer from the Fourier Transform to the Scanning Grating Instrument}

The above 5 methods were also tested for the calibration transfer between the Fourier transform and scanning grating instruments. For the SB technique, the slope and bias (intercept) of the regression line were 0.29 and 63.20 , respectively, by calculation on the standardization set of 30 samples. Predictions of validation samples obtained for the slave 2 instrument were then corrected by the 2 factors. For the OSC technique, the accuracy attenuation of the NIRS model developed on the corrected spectrum of the calibration set was negligible. The best number of PLS terms of calibration corrected by OSC was also 7 , and results of the correlation coefficient (0.89) and RMSEC (5.41 g/kg of $\mathrm{DM}$ ) were gained that were close to the calibration results before OSC. For the PDS method, the optimum window size of 5 , with the best value of RMSEP and bias was ultimately chosen.

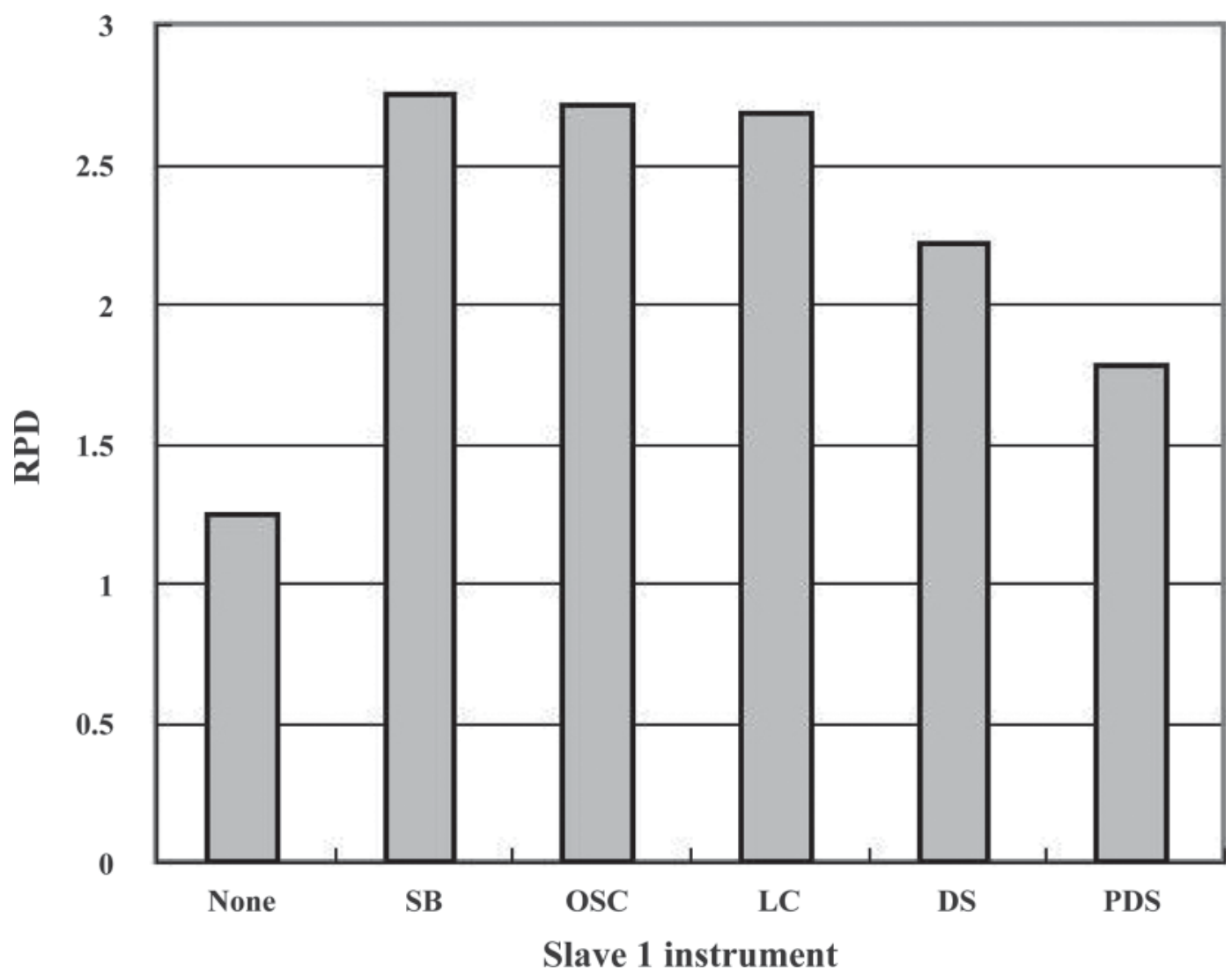

Figure 5. Relative prediction deviation (RPD) values of the slave 1 instrument (Antaris; Thermo Nicolet Corp., Madison, WI) after being transferred by different methods. $\mathrm{SB}=$ slope/bias correction; OSC = orthogonal signal correction; LC = local centering; DS = direct standardization; PDS = piecewise direct standardization. 
Table 4. Results of the slave 2 instrument (NIRSystems 6500; Foss A/S, Hillerød, Denmark) after being transferred by different methods

\begin{tabular}{lccccrr}
\hline & \multicolumn{7}{c}{ Method $^{2}$} \\
\cline { 2 - 7 } Parameter $^{1}$ & None & SB & OSC & \multicolumn{1}{c}{ LC } & \multicolumn{1}{c}{ DS } & \multicolumn{1}{c}{ PDS } \\
\hline RMSEP & 22.28 & 15.06 & 8.45 & 21.54 & 17.47 & 19.19 \\
Bias & 6.75 & -2.49 & 0.27 & -3.62 & 3.77 & -3.52 \\
ADS & 0.31 & - & 0.08 & 0.21 & 0.21 & 0.14 \\
P $_{\text {ADS }} / \%$ & - & - & 93.34 & 54.11 & 54.11 & 79.60 \\
\hline
\end{tabular}

${ }^{1} \mathrm{RMSEP}=$ root mean square error of prediction; ADS $=$ average diversity of spectra; $\mathrm{P}=$ loading.

${ }^{2} \mathrm{SB}=$ slope/bias correction; OSC $=$ orthogonal signal correction; $\mathrm{LC}=$ local centering; $\mathrm{DS}=$ direct standardization; PDS = piecewise direct standardization.

The results of prediction errors, $\mathrm{ADS}$, and $\mathrm{P}_{\mathrm{ADS}}$ for the slave 2 instrument after being transferred by different methods are shown in Table 4. Figure 6 shows the results of the RPD value.

The predictions on spectra of validation samples collected on the slave 2 instrument using the calibration model established on the master instrument were improved by all of the methods. Similar to the results of the slave 1 instrument, all of the prediction errors derived by SB, OSC, LC, DS, and PDS methods were decreased to different degrees for the slave 2 instrument compared with results of the non-transfer technique used. The most significant efficiency was found for the OSC method; the RMSEP and bias values after being transferred was much lower than that of the other 4 methods. The diversity of spectra of validation samples collected from the master and slave 2 instrument was also lessened after being transferred by the OSC, LC, $\mathrm{DS}$, and PDS methods, and the $\mathrm{P}_{\mathrm{ADS}}$ value was high $(93.34 \%)$ for the OSC method, moderate for the PDS

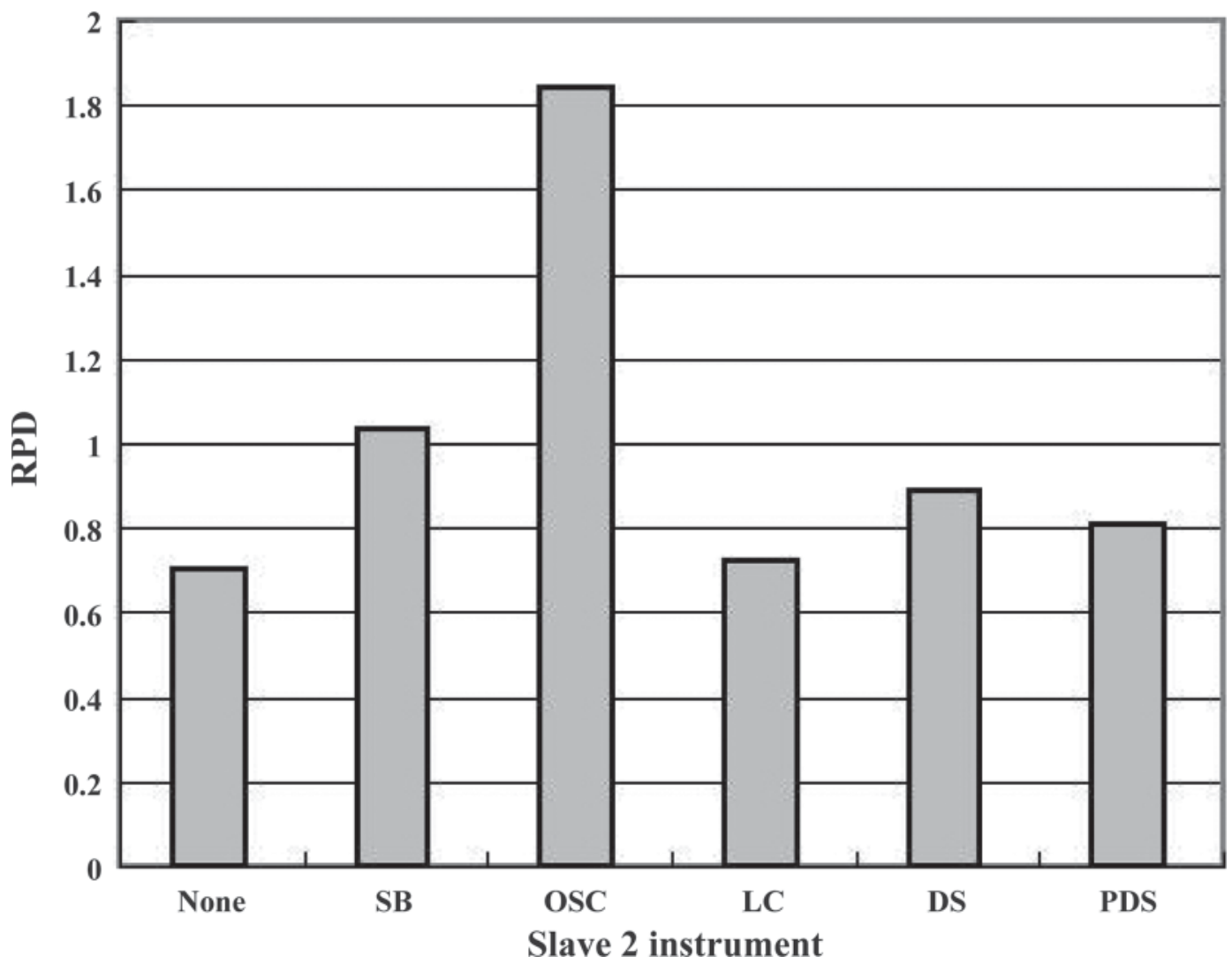

Figure 6. Relative prediction deviation (RPD) values of the slave 2 instrument (NIRSystems 6500; Foss A/S, Hillerød, Denmark) after being transferred by different methods. $\mathrm{SB}=$ slope/bias correction; OSC $=$ orthogonal signal correction; $\mathrm{LC}=$ local centering; $\mathrm{DS}=$ direct standardization; PDS = piecewise direct standardization. 
method, and lower for both the LC and DS method. The subtracted spectra of validation set before and after transfer of the OSC method for the master and the slave 2 instruments is shown in Figure 7, which proved that the absorbance difference between spectra measured on the 2 instruments was greatly decreased after OSC.

Further evaluation by the RPD value suggested that prediction transferred by the OSC method was moderately useful, with an RPD value of 1.84 , among values between 1.75 and 2.25 (Figure 6). Results showed that the RPD values of the SB, LC, DS, and PDS techniques were too low (lower than 1.50) to be suitable for practical applications. Although the prediction errors for slave instruments are always expected to be as low as those for the master system, some holdbacks exist for 2 instruments with great differences, just like the Fourier transform and scanning grating instrument in the present study. However, it is known that the calibrations could be improved gradually by database expansion, more active chemometrics, and other efficient methods. It should be possible to improve the predictions for a slave instrument transferred by OSC method by more advanced models established on the master system. We are making efforts on this now and hope to transfer the predictions with the optimizing method derived from this study.
It could be concluded that the OSC technique gave appreciate results not only for calibration transfer between the 2 Fourier transform instruments, but also for calibration transfer from the Fourier transform to the scanning grating instrument. This means it could work well when the instrumental differences were simple or systematic shifts in spectral data were measured on slave instruments, and when the instrumental differences were more complex and variable as well. The OSC method was originally introduced by Wold et al. (1998) to remove systematic variation from the response matrix (absorption) that is unrelated, or orthogonal, to the property matrix (concentration). Later, it was designed to remove extraneous variance from $X$ that is unrelated to $Y$ by finding directions in $X$ that describe large variances while being orthogonal to $Y$ (Feudale et al., 2002). Based on this reasoning, as in this study, OSC has been used successfully for calibration transfer between instruments (Sjöblom et al., 1998; Geladi et al., 1999; Greensill et al., 2001).

It was also proven that SB and LC techniques performed well in this study for calibration transfer between 2 Fourier transform instruments, whereas pooled results were obtained for both of them for calibration transfer from the Fourier transform to the scanning grating instrument. This was consistent with many studies in the literature; SB and LC methods worked

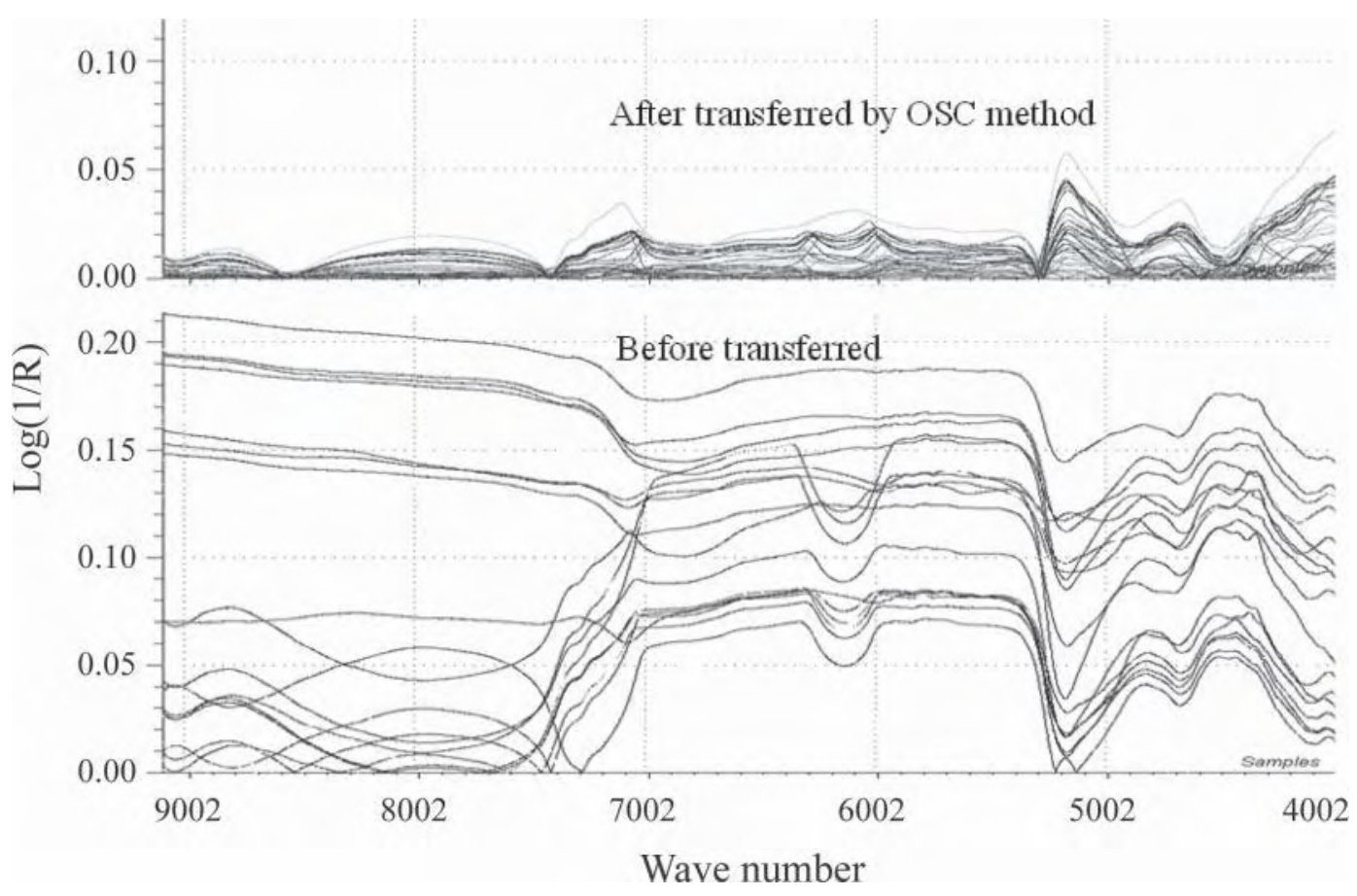

Figure 7. The subtracted spectra of the validation set before and after being transferred by the orthogonal signal correction (OSC) method for the slave 2 instrument (NIRSystems 6500; Foss A/S, Hillerød, Denmark). $\mathrm{R}$ = reflectance ratio of light. 
well when the 2 instruments were similar and the differences were simple or systematic (Bouveresse et al., 1996; Sjöblom et al., 1998; Macho et al., 2001). Although in the present study, the SB technique performed slightly better than the LC method, it was simpler to perform the LC method. For more complicated situations, a larger variation in the content of the active ingredient of the samples and more samples may be needed for $\mathrm{SB}$, whereas for the LC method, only a few transfer samples are required and they do not have to vary in content (Bergman et al., 2006).

\section{CONCLUSIONS}

The spectra obtained with different types of instruments or different configurations with the same type of instrument were different and not solely due to the differences in offset. The spectra were clearly grouped into clusters corresponding to the 3 studied NIRS instruments for PCA analysis. The differences in spectra between the 2 Fourier transform instruments were obviously smaller than the differences in spectra between the Fourier transform instrument and the scanning grating instrument. In the present study, it is possible to transfer calibrations for silage CP detection between instruments with different configurations using the same type or different types of instruments. No significant differences could be found for the predictions achieved from the master instrument and the ones achieved from the slave instruments with different configurations after transfer using the best methods. However, for calibration transfer between instruments of different types, the predictive ability of the calibration established on the master instrument was loosened to a certain extent. The SB, OSC, and LC techniques were successful in transferring NIRS calibration for silage detection between the 2 Fourier transform instruments; the SB and OSC methods performed slightly better than the LC method. The best result was given for OSC ahead of the other 4 techniques for transferring calibrations between Fourier transform and scanning grating instruments, and it was evaluated as moderately useful. Further work should be carried out to improve the predictions for slave instruments transferred by the OSC method by more advanced models established on the master system.

\section{ACKNOWLEDGMENTS}

The authors acknowledge the financial support of the agro-scientific research project (Contracts 201003063 and 200903009) in the public interest, Ministry of Agriculture (MOA), China. Special thanks go to Foss A/S and Nicolet Corp. China Beijing Representative Office for the supply of NIRS instruments.

\section{REFERENCES}

AOAC. 2001. Official Methods of Analysis. Method 2011.11. Protein (crude) in animal feed, forage (plant tissue), grain, and oilseeds. Association of Official Analytical Chemists, Gaithersburg, MD.

Bergman, E.-L., H. Brage, M. Josefson, O. Svensson, and A. Sparén. 2006. Transfer of NIR calibrations for pharmaceutical formulations between different instruments. J. Pharm. Biomed. Anal. 41:89-98.

Blank, T. B., S. T. Sum, S. D. Brown, and S. L. Monfre. 1996. Transfer of near-infrared calibrations without standards. Anal. Chem. 68:2987-2995

Bouveresse, E., C. Hartmann, D. L. Massart, I. R. Last, and K. A. Prebble. 1996. Standardization of near-infrared spectrometric instruments. Anal. Chem. 68:982-990.

Cozzolino, D., A. Fassio, and A. Gimenez. 2001. The use of near-infrared reflectance spectroscopy to predict the composition of whole maize plants. J. Sci. Food Agric. 81:142-146.

De Boever, J. L., B. G. Cottyn, D. L. De Brabander, J. M. Vanacker, and Ch. V. Boucqué. 1997. Prediction of the feeding value of maize silages by chemical parameters, in vitro digestibility and NIRS. Anim. Feed Sci. Technol. 66:211-222.

Fearn, T. 2001. Standardisation and calibration transfer for near infrared instruments: A review. J. Near Infrared Spectrosc. 9:229-244.

Feudale, R. N., N. A. Woody, H. Tan, A. J. Myles, S. D. Brown, and J. Ferre. 2002. Transfer of multivariate calibration models: A review. Chemom. Intell. Lab. Syst. 64:181-192.

Geladi, P., H. Barring, E. Dabakk, J. Trygg, H. Antti, S. Wold, and B. Karlberg. 1999. Calibration transfers for predicting lake-water $\mathrm{pH}$ from near infrared spectra of lake sediments. J. Near Infrared Spectrosc. 7:251-264.

Greensill, C. V., P. J. Wolfs, C. H. Spiegelman, and K. B. Walsh. 2001. Calibration transfer between PDA-based NIR spectrometers in the NIR assessment of melon soluble solid content. Appl. Spectrosc. 55:647-653.

Hein, P. R. G., J. T. Lima, and G. Chaix. 2009. Robustness of models based on near infrared spectra to predict the basic density in $E u$ calyptus urophylla wood. J. Near Infrared Spectrosc. 17:141-150.

Koehler, F. W., G. W. Small, R. J. Combs, R. B. Knapp, and R. T. Kroutil. 2000. Calibration transfer algorithm for automated qualitative analysis by passive Fourier transform infrared spectrometry. Anal. Chem. 72:1690-1698.

Leion, H., S. Folestad, M. Josefson, and A. Sparén. 2005. Evaluation of basic algorithms for transferring quantitative multivariate calibration between scanning grating and FT NIR spectrometers. J. Pharm. Biomed. Anal. 37:47-55.

Liu, X., and L. J. Han. 2006. Prediction of chemical parameters in maize silage by near infrared reflectance spectroscopy. J. Near Infrared Spectrosc. 14:333-339.

Liu, X., L. Han, Z. Yang, and Ch. Xu. 2008. Prediction of silage digestibility by near infrared reflectance spectroscopy. J. Anim. Feed Sci. 17:631-639.

Lorber, A., K. Faber, and B. R. Kowalski. 1996. Local centering in multivariate calibration. J. Chemometr. 10:215-220.

Macho, S., A. Rius, M. P. Callao, and M. S. Larrechi. 2001. Monitoring ethylene content in heterophasic copolymers by near-infrared spectroscopy: Standardization of the calibration model. Ana1. Chim. Acta 445:213-220.

Malley, D. F., E. Ben-Dor, and P. D. Martin. 2004. Application in analysis of soils. Pages 729-784 in Near Infrared Spectroscopy in Agriculture. C. Roberts, J. Workman, and J. B. Reeves III, ed. American Society of Agronomy, Soil Science Society of America, and Crop Science Society of America, Madison, WI.

Melchinger, A. E., G. A. Schmidt, and H. H. Geiger. 1986. Evaluation of near infra-red reflectance spectroscopy for predicting grain and stover quality traits in maize. Plant Breed. 97:20-29. 
Moe, A. J., and S. B. Carr. 1985. Laboratory assays and near-infrared reflectance spectroscopy for estimates of feeding value of corn silage. J. Dairy Sci. 68:2220-2226.

Murray, I. 1993. Forage analysis by near infrared spectroscopy. Pages 285-312 in Sward Measurement Handbook. 2nd ed. A. Davies, R. D. Baker, S. A. Grant, and A. S. Laidlaw, ed. The British Grassland Society, Kenilworth, Warwickshire, UK.

Savitzky, A., and M. J. E. Golay. 1964. Smoothing and differentiation of data by simplified least-squares procedures. Anal. Chem. 36:1627-1639

Schimleck, L. R., C. Mora, and R. F. Daniels. 2003. Estimation of the physical wood properties of green Pinus taeda radial samples by near infrared spectroscopy. Can. J. For. Res. 33:2297-2305.

Shenk, J. S., and M. O. Westerhaus. 1991. New standardization and calibration procedures for NIRS analytical systems. Crop Sci. 31:1694-1696.

Shenk, J. S., and M. O. Westerhaus. 1993. Comments on standardisation: Part 2. NIR News 4:13-15.
Sjöblom, J., O. Svensson, M. Josefson, H. Kullberg, and S. Wold. 1998 An evaluation of orthogonal signal correction applied to calibration transfer of near infrared spectra. Chemom. Intell. Lab. Syst. $44: 229-244$

Sørensen, L. K. 2004. Prediction of fermentation parameters in grass and corn silage by near infrared spectroscopy. J. Dairy Sci. $87: 3826-3835$.

Wang, Y., D. J. Veltkamp, and B. R. Kowalski. 1991. Multivariate instrument standardization. Anal. Chem. 63:2750-2756.

Williams, P. C., and D. C. Sobering. 1993. Comparison of commercial near infrared transmittance and reflectance instruments for analysis of whole grains and seeds. J. Near Infrared Spectrosc. 1:25-33.

Wold, S., H. Antti, F. Lindgren, and J. Ohman. 1998. Orthogonal signal correction of near-infrared spectra. Chemom. Intell. Lab. Syst. $44: 175-185$. 